\title{
FLOW STRUCTURES IN THREE-DIMENSIONAL FISH-LIKE SWIMMING
}

\author{
Xing Zhang \\ LNM, Institute of Mechanics, \\ Chinese Academy of Sciences \\ Beijing, China
}

\author{
Shizhao Wang \\ LNM, Institute of Mechanics, \\ Chinese Academy of Sciences \\ Beijing, China
}

\author{
Guowei He \\ LNM, Institute of Mechanics, \\ Chinese Academy of Sciences \\ Beijing, China
}

\begin{abstract}
The swimming of a fish-like body is numerical simulated. The wake structures consist of a series of hairpin-like vortices braided together. The caudal fins generated vorticity interacts constructively with the body-bounded vorticity.
\end{abstract}

\section{INTRODUCTION}

The unsteady undulatory swimming of fish is expected to have a high hydrodynamic performance. A recent review on this subject has been given by Bandyopadhyay [1]. Closely related to this high hydrodynamic performance are the flow structures. These flow structures can be considered as the combination of separated boundary layers, vortices generated by dorsal fins or finlets or caudal fins, and their interactions. Studies on the flow structures are helpful to identify the principle mechanisms of vorticity control employed in fish swimming.

Numerical simulation has an advantage in obtaining the whole-field flow information, and is suitable for investigating flow structures in fish swimming. Previous numerical studies mostly focused on the two-dimensional cases. The aspect-ratio of the fins/tail of fish is low, and the three-dimensional effects may play an important role in fish swimming. In this work, we numerically simulate the swimming of a three dimensional fishlike body. The hydrodynamics of fish-like swimming and the vortex structures around the fish-like body and in the wake are investigated.

\section{MODELS AND METHODS Geometry and dynamics}

In the present work, a body shape representing a tuna is employed. The parameters of the model are taken from Zhu [2] with a little modification. The geometry of the body is shown in figure 1.

The wave-like motion of body is described as (in the bodyfixed coordinate system)

$$
\begin{aligned}
& y(x, t)=a(x) \sin \left(k_{\omega} x-\omega t\right) \\
& a(x)=c_{1} x+c_{2} x^{2}
\end{aligned}
$$

where $k_{\omega} \equiv 2 \pi / \lambda$ is the wave number, corresponding to wavelength $\lambda, \omega$ is the circular frequency of oscillation, $a(x)$ is defined by the adjustable parameters $c_{1}$ and $c_{2}$, representing the smoothly varying of the amplitude of the waving body.

The caudal fins are allowed to undergo a pitch motion around the front point. The pitching motion is taken as:

$$
\theta=\alpha \sin \left(k_{w} x_{p}-\omega t-\varphi\right),
$$

where $\alpha$ is the amplitude of the pitch angle and. $\varphi$ the phase lag between the undulation and pitch.

The parameters in present simulation is taken as: $\lambda=1.22, \quad \omega=2 \pi, \quad c_{1}=0.0, \quad c_{2}=0.155, \quad \alpha=\pi / 6.0$, $\varphi=\pi / 2.0$.

\section{Governing equations and boundary conditions}

The equations governing the flow around the fish-like body are the three-dimensional, unsteady incompressible NavierStokes equations

$$
\begin{aligned}
& \partial u_{i} / \partial x_{i}=0 \\
& D u_{i} / D t=-\partial p / \partial x_{i}+1 / \operatorname{Re}\left(\partial^{2} u_{i} / \partial x_{j} \partial x_{j}\right)+f_{i}
\end{aligned}
$$

where $u_{i}$ and $p$ are the velocity and pressure respectively. Reynolds number is defined as $\operatorname{Re}=\omega A^{2} / v, A$ the flapping amplitude of the trailing edge of the tail and $v$ the kinematic viscosity.

Non-slip boundary condition is applied on the surface of the fish-like body and stress-free boundary conditions are applied on the boundaries of the computational domain. 


\section{Numerical method}

The Navier-Stokes equations are solved on a Cartesian grid using Immersed Boundary (IB) Method to simulate the swimming of fish-like body. The unstructured, triangular mesh is used to discrete and track the surface of the fish body. The direct forcing is used to represent the effect of the complex surface of the fish to the flow, and the IB Method is used in the framework of the discrete stream-function approach. The forcing term to impose the non-slip boundary condition is determined implicitly (by solving a linear system).

The technical details of this numerical methodology and some benchmark validations can be found in [3].

\section{RESULTS}

\section{Wake structures}

The wake structures of the swimming of fish-like body are shown in Figure 2. The vortical structures in the wake are visualized use the second invariant value of the tensor of velocity gradient [4].

The structures range in a single row and remain confined within a relatively narrow strip that is centered about the horizontal axis of the fish body. The wake structures consist of a series of hairpin-like vortices braided together such that the legs of each vortex are attached to the head of the preceding one.

\section{Vorticity control mechanism}

Fish is suspected to be smart enough to alter the position and the strength of oncoming body-bounded vortices, thereby affecting upstream velocity of the caudal fins to improve the hydrodynamic performance. The interactions of body-bounded vortices and caudal fin generated vortices are shown in Figure3.

The process of constructive interference can be identified in Figure 3. The caudal fins generated vorticity interacts constructively with the oncoming body generated vorticity, resulting in the generation of a strong vortex street in the downstream of the caudal fins.

\section{Discussion}

An important characteristic of fish swimming is the unsteady self-propelled locomotion. The flows around a selfpropelled fish may have a major difference from that of a towed model. The numerical simulation of the swimming of a three dimensional self-propelled fish-like body may be better to understand the mechanism of vorticity control and flow structures characteristics.

\section{CONCLUSION}

The swimming of a three-dimensional fish-like body is numerically simulated. The fish-like body undergoes a prescribed undulation in the uniform upstream flow. The wake structures under the present conditions show a single-row wake with of a series of hairpin-like vortices braided together. The caudal fins generated vorticity interacts constructively with the oncoming body-bounded vorticity.

The flow structures are related to the morphology, kinematics and swimming Reynolds number. A more detail analysis of the relations between the flow structures and these parameters will be conducted in the future work.

\section{ACKNOWLEDGMENTS}

This work was supported by Chinese Academy of Sciences under the Innovative Projects "Multi-scale modeling and simulation in complex systems" (KJCX-SW-L08)and "Mathematical modeling of complex system" (KJCX3-SYW-S01)

\section{REFERENCES}

[1] Bandyopadhyay, P., 2009, "Swimming and flying in nature-the route toward applications: the freeman scholar lecture". J Fluids Eng, 131, 031801.

[2] Zhu, Q., Wolfgang, M., and Triantafyllou, M., 2002, "Threedimensional flow structures and vorticity control in fish-like swimming". J Fluid Mech, 468, pp.1-28.

[3] Zhang X., "An immersed boundary method using the discrete streamfunction approach with an implicit forcing". 62nd Annual Meeting of the APS Division of Fluid Dynamics, November 2224, 2009. Minneapolis, Minnesota, USA. AbstractID: BAPS.2009.DFD.EL.5.

[4] Hunt, J., Wray,A. and Moin, P., 1988, "Eddies, streams, and convergence zones in turbulent flows". In Its Studying Turbulence Using Numerical Simulation Databases, 2, Proceedings of the 1988 Summer Program (SEE N89-24538 1834), pp.193-208.

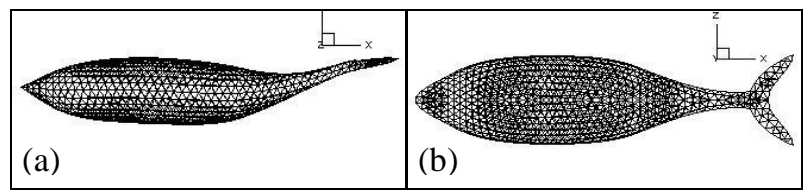

Figure 1. Scheme of the fish-like model. (a) top view; (b) side view

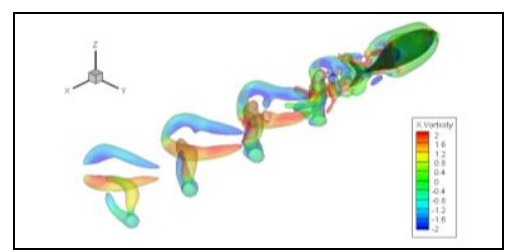

Figure 2. Wake structures for the swimming of fish-like body. The isosurfaces of the second invariant value of the tensor of velocity gradient are plotted. The iso-surfaces are colored with the values of stream-wise vorticity. The structures consist of a series of inverted hairpin-like vortices that are braided together such that the legs of each vortex are attached to the head of the preceding one.

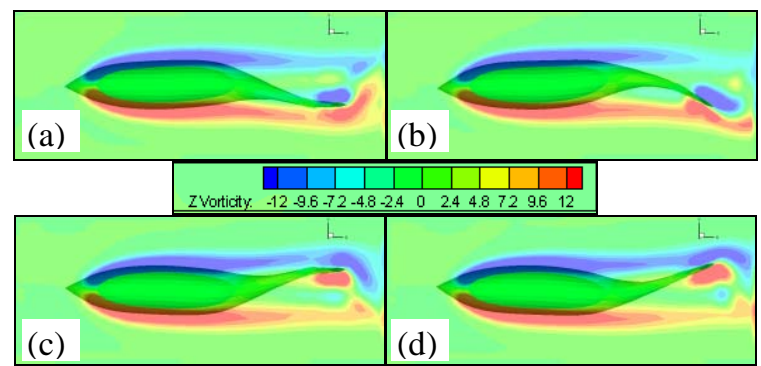

Figure 3. The interactions of body bounded vortices and caudal fin generated vortices at different time. The z-vorticity in x-o-y plane at $\mathrm{z}$ $=0.1$ is plotted. (a) separation of the positive caudal fin leading edge vortex; (b) increasing of the negative caudal fin leading edge vortex; (c) separation of the negative caudal fin leading edge vortex; (d) increasing of the positive caudal fin leading edge vortex. 